Ganzheitsmedizin

Swiss Journal of Integrative Medicine

\section{Fortbildung}

Schweiz Z Ganzheitsmed 2010;22:334 DOI: $10.1159 / 000322353$
Published online: November 22,2010

\author{
Jean-Michel Jeannin
}

\section{Einleitung}

Der Kakaobaum (Theobroma cacao) stammt aus dem tropischen Regenwald des Amazonas- und des Orinoco-Beckens [1]. Der Baum wird bis zu $8 \mathrm{~m}$ hoch [2]. Die höchstens $1 \mathrm{~cm}$ grossen hübschen weissen Blüten zeigen sich das ganze Jahr über. Sie wachsen in Büscheln direkt am Stamm oder an starken Seitenästen. Aus den Blüten entwickeln sich die gurkenförmigen, 15-20 cm langen Früchte. Sie enthalten bis zu 50 Samen (Bohnen) [1]. Die Kakaobohnen werden sowohl arzneilich als auch als Ausgangsstoff für Lebensmittel verwendet. Belegt ist auch die medizinische Verwendung der Blüten [3].

\section{Verwendung als Heilpflanze}

Die ältesten Zeugnisse für die Verwendung des Kakaos stammen aus der Zeit der Entdeckung. Sowohl die

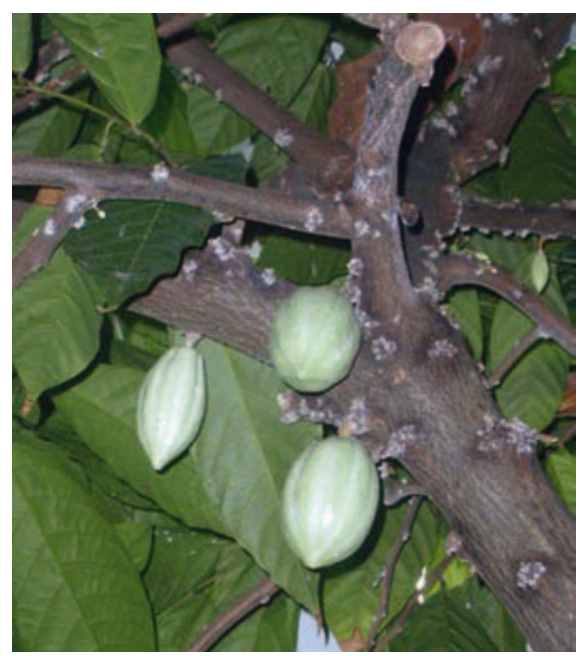

Abb. 1. Theobroma cacao: Reifende Früchte. Tropisches Gewächshaus der Universität Basel, 2010.

\title{
Exotische Heilpflanzen (6)
}

\section{Theobroma cacao L. (Sterculiaceae, neu: Malvaceae)}

Maya als auch die Azteken betrachteten den Kakao als göttliche Gabe. In der aztekischen Kultur war der Kakao dem Adel, den hohen Militärs und den Priestern vorbehalten. Er wurde auch rituell verwendet und Menschen, die geopfert werden sollten, zur Stärkung verabreicht. Die Azteken verabreichten ein Getränk aus Kakao zur Behandlung von Magen-DarmBeschwerden. Zubereitungen, die Kakaobohnen enthielten, wurden gegen kindlichen Durchfall gegeben. Ein mit den Blüten parfümiertes Bad wurde zur Behandlung von Erschöpfungszuständen verordnet. In der Alten Welt wurde Kakao oder Schokolade als Stärkungs- und Aufbaumittel, als Stimulans oder als Beruhigungsmittel und zur Darmregulierung verabreicht [3].

Die Azteken benutzten die Kakaobohnen als Währung und horteten sie auch als Währungsreserve; die Kakaobutter ergibt eine natürliche Grundmasse für Suppositorien. Geröstete Kakaobohnen sind die Grund-

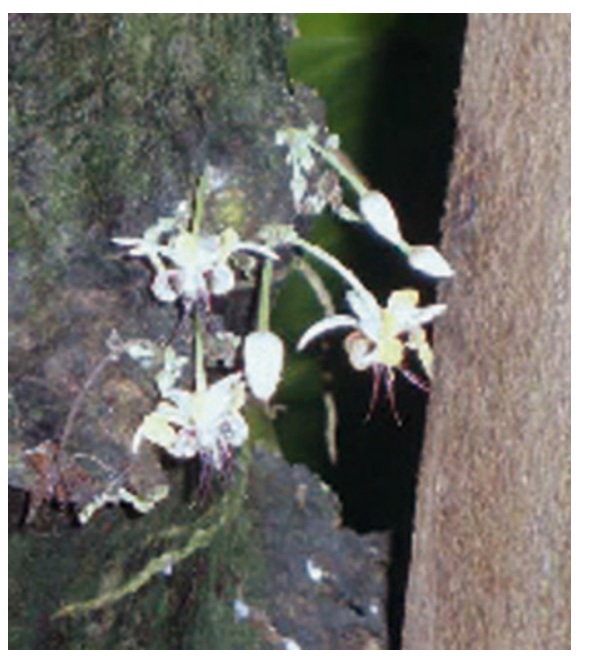

Abb. 2. T. cacao: Blüten. Tropisches Gewächshaus der Universität Basel, 2010. lage zahlloser Frühstücksgetränke und Schokoladenprodukte.

\section{Phytochemie}

Die Kakaobohnen enthalten Polyphenole in hohen Konzentrationen. Rund $37 \%$ der Polyphenole sind Flavan-3ole, 4\% Anthocyanine und 58\% Proanthocyanine [4]. Als pharmakologisch wirksame Substanzen enthalten die Bohnen die zwei Methylxanthine Theobromin und Coffein [2]. In den Sprossen wurden 9,75\% Catechine und 2,24\% Coffein gefunden, in den Blättern jeweils 5,25\% und 1,33\% [5].

\section{Aktuelle Forschung}

Kakao und Schokolade verringern das Risiko von Herz-Kreislauf-Krankheiten [6]. Theobromin hat das Potenzial zu einem Antitussivum [7].

\section{Literatur}

1 Stöcklin J: Kakaobaum. Text zur Ausstellung «Wegzehrung». Universität Basel, 1996.

2 Cocoa (Cacao); in Leung AY, Foster S: Encyclopaedia of Common Natural Ingredients Used in Food, Drugs and Cosmetics, ed 2. New York, John Wiley \& Sons, 1996.

3 Dillinger TL, et al: Food of the gods: cure for humanity? A cultural history of the medicinal and ritual use of chocolate. J Nutr 2000;130:2057S-2072S.

4 Rusconi M, Conti A: Theobroma cacao L., the food of the gods: a scientific approach beyond myths and claims. Pharmacol Res 2010;61:5-13.

5 Osman H, et al: Extracts of cocoa (Theobroma cacao L.) leaves and their antioxidation potential. Food Chem 2004;86:41-46.

6 Ding EL, et al: Chocolate and prevention of cardiovascular disease: a systematic review. Nutr Metab 2006;3:2.

7 Usmani OS, et al: Theobromine inhibits sensory nerve activation and cough. FASEB J 2005;19:231-233.

\section{KARGER}

Fax +497614520714 Information@Karger.de www.karger.com (c) 2010 S. Karger GmbH, Freiburg
Dipl. med. biol. Jean-Michel Jeannin

Holeestrasse 43, 4054 Basel, Schweiz

Tel. +41 61-4215991, Fax -4230313

jmjeannin@dataworks.ch 